\title{
BMJ Open Effect of bicyclol on blood biomarkers of NAFLD: a systematic review and meta-analysis
}

\author{
$\mathrm{Hu}$ Li (D) , ${ }^{1,2}$ Nan-Nan Liu, ${ }^{1}$ Zong-Gen Peng ${ }^{1,3}$
}

To cite: Li H, Liu N-N, Peng ZG. Effect of bicyclol on blood biomarkers of NAFLD: a systematic review and meta-analysis. BMJ Open 2020;10:e039700. doi:10.1136/ bmjopen-2020-039700

\section{- Prepublication history and} additional material for this paper are available online. To view these files, please visit the journal online (http://dx.doi. org/10.1136/bmjopen-2020039700).

Received 23 April 2020 Revised 21 July 2020 Accepted 20 October 2020

\section{Check for updates}

(c) Author(s) (or their employer(s)) 2020. Re-use permitted under CC BY-NC. No commercial re-use. See rights and permissions. Published by BMJ.

${ }^{1}$ CAMS Key Laboratory of Antiviral Drug Research, Institute of Medicinal Biotechnology, Chinese Academy of Medical Sciences and Peking Union Medical College, Beijing, China ${ }^{2}$ Key Laboratory of Biotechnology of Antibiotics, The National Health and Family Planning Commission (NHFPC), Institute of Medicinal Biotechnology, Chinese Academy of Medical Sciences and Peking Union Medical College, Beijing, China

${ }^{3}$ Beijing Key Laboratory of Antimicrobial Agents, Institute of Medicinal Biotechnology, Chinese Academy of Medical Sciences and Peking Union Medical College, Beijing, China

Correspondence to Professor Zong-Gen Peng; pumcpzg@126.com

\section{ABSTRACT}

Objective Non-alcoholic fatty liver disease (NAFLD) is a global epidemic without effective therapeutic agents in the clinic. This meta-analysis aimed to assess the efficacy of the marketed hepatoprotectant bicyclol at improving blood biomarkers in patients with NAFLD.

Design Electronic databases were searched for randomised controlled trials (RCTs) published up to August 2020 using bicyclol to treat NAFLD. The risk of bias, quality of evidence and publication bias were evaluated. Blood biomarkers, including alanine transaminase (ALT), aspartate aminotransferase (AST), total bilirubin (TBIL), triglyceride (TG) and total cholesterol (TC), were analysed using Review Manager V.5.3 software. Outcomes with significant heterogeneity $\left(1^{2} \geq 75 \%\right)$ were divided into the bicyclol monotherapy subgroup and combination treatment subgroup.

Results Twelve RCTs involving 1008 patients were finally included. No serious adverse events were reported in the bicyclol-treated groups. The total effective rate of bicyclol intervention for NAFLD was significantly higher than that of the control group. The decreases in the levels of AST (mean difference (MD) $=-15.20 ; 95 \% \mathrm{Cl}-20.51$ to $\left.-9.90 ; l^{2}=74 \%\right)$, TBIL (MD $=-1.72 ; 95 \% \mathrm{Cl}-2.72$ to $\left.-0.72 ; \mathrm{I}^{2}=0 \%\right)$ and TC (MD $=-0.52 ; 95 \% \mathrm{Cl}-0.70$ to $-0.34 ; I^{2}=67 \%$ ) treated by bicyclol were significantly higher than those in the control group. When a high heterogeneity existed $\left(I^{2} \geq 75 \%\right)$, subgroup analyses were conducted and revealed significantly decreased ALT levels $\left(\mathrm{MD}=-34.07 ; 95 \% \mathrm{Cl}-36.70\right.$ to $-31.43 ; \mathrm{I}^{2}=0 \%$ ) merely in the bicyclol monotherapy subgroup, while TG level (MD $=-0.39 ; 95 \% \mathrm{Cl}-0.45$ to $-0.33 ;\left.\right|^{2}=0 \%$ ) was decreased in the bicyclol combination therapy subgroup.

Conclusions The study presents the evidence of bicyclol monotherapy and/or combination therapy for improving liver function and blood lipid biomarkers in patients with NAFLD. This preliminary study predicts that bicyclol might be an alternative drug for NAFLD therapy in the future.

\section{INTRODUCTION}

Non-alcoholic fatty liver disease (NAFLD) is the most common spectrum of liver diseases typically ranging from non-alcoholic fatty liver (NAFL) to non-alcoholic steatohepatitis (NASH). ${ }^{1}$ Benign and reversible NAFL is merely characterised by excessive lipid droplet deposition in hepatocytes, while NASH is a more aggressive condition characterised

\section{Strengths and limitations of this study}

This systematic review is the first to determine the effect of bicyclol on blood biomarkers of patients with non-alcoholic fatty liver disease (NAFLD).

- This study provides preliminary evidence that bicyclol might be efficacious for treatment of patients with NAFLD.

- The limitation of this meta-analysis is the low quality of the existing studies, and the results of this study only apply to China because bicyclol has not been approved in Europe and North America.

by inflammatory infiltrates, visible cellular injury and possible progression to, or accompanied by, fibrosis and cirrhosis. ${ }^{2}$ NAFLD is closely related to the high incidence of metabolic syndrome, cardiovascular disease, type 2 diabetes mellitus (T2DM) and advanced liver diseases. ${ }^{13}$ Currently, the prevalence of NAFLD worldwide is up to $25 \%$, with the highest prevalence of $32 \%$ reported in the Middle East and $31 \%$ in South America, and even the lowest prevalence in Africa was estimated to be $14 \% .{ }^{4}$ Worse still, the prevalence of NAFLD worldwide is presumed to be increasing. ${ }^{5}$ There are no admitted therapeutic agents from international societies for treating NAFLD, except for lifestyle changes. ${ }^{6-8}$ However, patients tend to exhibit poor adherence to this important intervention. ${ }^{9}$ Recently, only one dual peroxisome proliferator-activated receptor- $\alpha / \gamma$ agonist saroglitazar magnesium has been approved for the treatment of NASH without cirrhosis in India. ${ }^{10}$ However, numerous potential agents, such as farnesoid $\mathrm{X}$ receptor agonists, apoptosis signal-regulated kinase 1 inhibitors and C-C chemokine receptor type 2/5 inhibitors, have entered different phases in clinical trials but presented limited or even no benefits. ${ }^{111} 12$ Therefore, new or complementary drugs for treating NAFLD are still urgently needed and this dilemma might persist for a long time. 
Bicyclol, a hepatoprotective and anti-inflammatory drug that has been approved in China since 2004, was used to treat increased levels of aminotransferases caused by various forms of chronic hepatitis mainly in Asian countries, while it has not been approved in Europe and North America. ${ }^{13}$ It is rather safe and suitable for longterm (more than 6 months) oral administration. ${ }^{13}$ Many preclinical animal experiments have confirmed its therapeutic effect in chemical-induced, immunological, fatty and drug-induced liver injury, as well as hepatic fibrosis caused by bile duct ligation, dimethylnitrosamine, bovine serum albumin or carbon tetrachloride. ${ }^{13-15}$ The detailed mechanisms of bicyclol involve the inhibition of hepatocyte apoptosis, stabilisation of mitochondrial or hepatocyte membranes, scavenging free radicals, increasing the expression of antioxidant genes and reducing lipid peroxide levels. ${ }^{14} 16$ Although liver histology and MRI have high accuracy for evaluating the liver fat content, ${ }^{17}$ liver function and blood lipid biomarkers, which mainly include alanine transaminase (ALT), aspartate aminotransferase (AST), total bilirubin (TBIL), triglyceride (TG) and total cholesterol (TC), are commonly used to evaluate the severity of NAFLD and the subsequent abnormal metabolism. ${ }^{18}{ }^{19}$ Relevant clinical and preclinical studies have reported the potential therapeutic role of bicyclol in NAFLD, ${ }^{20}{ }^{21}$ however, its effect on noninvasive blood biomarkers in patients with NAFLD has not been precisely confirmed due to insufficient sample sizes and the low quality of studies. Hence, this metaanalysis aimed to evidence the effect of bicyclol on blood biomarker levels in patients with NAFLD through synthesising the clinical data using bicyclol monotherapy alone or in combination with other drugs to treat NAFLD, and to preliminarily predict its clinical efficacy in the future.

\section{METHODS}

The data included in this meta-analysis were derived from previously published clinical studies, all of which were conducted in China. The study protocol was confirmed by all authors before data collection. Our protocol has been registered at the International Platform of Registered Systematic Review and Meta-Analysis Protocols (INPLASY). The registration number is INPLASY202080017 (DOI number is 10.37766/inplasy2020.8.0017, https://inplasy. com/inplasy-2020-8-0017/). We used analytical methods recommended in the Cochrane Handbook for Systematic Reviews of Interventions ${ }^{22}$ and reported this study following the Preferred Reporting Items for Systematic Reviews and Meta-Analyses checklist. ${ }^{23}$

\section{Search strategy}

Studies up to August 2020 were searched in PubMed, Embase, Cochrane Library and Chinese databases, including the China National Knowledge Infrastructure database, the WeiPu (VIP)-Chinese scientific and technological journal database, and the Wanfang digital periodical full-text database. Search terms were ('Non alcoholic Fatty
Liver Disease' OR 'NAFLD' OR 'nonalcoholic fatty liver' OR 'non-alcoholic fatty liver' OR 'Nonalcoholic Steatohepatitis' OR 'Nonalcoholic Steatohepatitides') AND ('bicyclol' OR '4,4'-bi-(1,3-benzodioxole)-5-carboxylic acid, 5'-(hydroxymethyl)-7,7'-dimethoxy-, 'methyl ester' OR '6-methoxycarbonyl-6-hydroxymethyl-2,3,2',3'-bis (me thylenedioxy)-4,4'- 'dimethoxybiphenyl') without other restrictions (online supplemental methods). Additional studies were hand-searched in Google Scholar and the reference lists of relevant articles.

\section{Inclusion and exclusion criteria}

The inclusion criteria were as follows: (1) Randomised controlled trials (RCTs); (2) Male and female patients diagnosed with NAFLD complicated with or without T2DM according to the corresponding guidelines; (3) An average baseline ALT level greater than 90 U/L (2 3 times the upper limit of normal values), ${ }^{24}$ while a TG level ranging from $2.5 \mathrm{mmol} / \mathrm{L}$ to $5 \mathrm{mmol} / \mathrm{L}$; and (4) Articles published in the English or Chinese language. The exclusion criteria were (1) Non-clinical studies, non-RCTs; (2) Studies examining patients with liver injury induced by drugs, viruses, alcohol, autoimmunity, primary biliary cholangitis, liver decompensation, malignancy or genetics; (3) Studies enrolling fewer than 20 subjects in each group, or the treatment time of less than 4 weeks; and (4) Studies without sufficient experimental data, such as case reports, reviews, conference abstracts, or a lack of sufficient biochemical indicators.

\section{Intervention measures}

The bicyclol monotherapy group (experimental group) was compared with groups treated with a lifestyle intervention (LSI) or another drug as a monotherapy (control group). Bicyclol combined with another medical treatment (experimental group) was compared with the corresponding medicine (control group). Other potential factors, such as LSIs were required to be consistent between the two groups.

\section{Outcome indicators}

Liver function indicators (ALT, AST and TBIL levels) and blood lipid parameters (TG and TC levels) were recorded. Adverse events, the anthropometric parameter body mass index (BMI), and the total effective rate, which was defined as the ratio of participants who have achieved significant decreases in blood biomarker levels (the decreased level of TC $>10 \%$ and TG $>20 \%$ ) and parameters of liver fat reduction under B-model ultrasonography among the included participants in the corresponding studies, were also analysed.

\section{Data extraction and quality assessment}

The outcome indicators from all included studies were independently extracted and checked by two authors (HL and NNL) to guarantee the accuracy of the data. The quality of RCTs, which was assigned as a 'high risk', 'low risk' or 'some concerns' for each item, was also assessed independently by 
two reviewers using the revised Cochrane risk of bias tool. ${ }^{25}$ Any discrepancies were resolved through discussion.

\section{Data analysis}

Review Manager V.5.3 software was used to analyse the data. ${ }^{26}{ }^{27}$ OR and pooled mean difference (MD) with the corresponding $95 \%$ CI were estimated for binary outcomes and continuous outcomes, respectively. Heterogeneities were evaluated using the $\chi^{2}$ and $\mathrm{I}^{2}$ statistics. ${ }^{26}$ When the outcome was homogeneous $\left(\mathrm{I}^{2}<50 \%\right.$ and $\left.\mathrm{P}>0.10\right)$, the fixed-effects model was used, and the random-effects model was used when the outcome was considered heterogeneous $\left(50 \% \leq \mathrm{I}^{2}<75 \%\right)$. When significant heterogeneity was observed (up to $75 \%$ ), a subgroup analysis was conducted according to bicyclol monotherapy and combination therapy, and if the $\mathrm{I}^{2}$ of the subgroup was still over $75 \%$, descriptive results were provided without pooling estimates. The statistical significance of differences between the experimental and control groups was set at $\mathrm{P}<0.05$. Publication bias was assessed only for comparisons with at least five studies using the funnel plot and its symmetry was evaluated using Egger's regression tests through Stata V.12.0 software. Significant publication bias was defined as $\mathrm{p}<0.100 .{ }^{28}$ Grading of evidence for the key comparisons was performed using the approach described by the Grading of Recommendations, Assessment, Development and Evaluation working group. ${ }^{22}$

\section{Patient and public involvement}

Patients and the public were not involved in this review.

\section{RESULTS}

\section{Study selection}

The whole flow chart of the data selection process is presented in figure 1 . Initially, 166 records were searched out, and 94 records were retained after duplicate exclusion. We then achieved 34 studies after screening the title and abstract, in which reviews, case reports, animal experiments, and studies with incongruent intervention measures and research orientation were excluded. After screening the full text, we excluded studies without appropriate samples, biochemical indicators, and baseline ALT and TG levels. One irrelevant study, which included patients with alcoholic fatty liver, was also excluded. Finally, 12 studies published in Chinese were included. ${ }^{29-40}$

\section{Characteristics, quality evaluation and publication bias of the included studies}

The characteristics of the included studies are presented in table 1. All the studies were conducted in China and published from 2005 to 2017, and the sample size ranged from 50 to 152 (median of 81). The total sample size is 1008 with 523 patients in the treatment group and 485 participants in the control group. The baseline values of patient outcome indicators were not different between the two groups.

The quality assessment of the included studies is shown in figure 2 according to the most recently revised Cochrane risk of bias tool (online supplemental table S1), in which one study applied the random number table, ${ }^{29}$ and other studies used randomisation but did not provide detailed methods. None of the studies reported the blinding condition or the plan of allocation and concealment. Additionally, all the studies had provided complete outcome data, without other predictable sources of bias.

The Egger's tests of funnel plots (online supplemental figure S1) for primary outcomes did not reveal significant publication bias among the blood biomarkers of AST ( 8 studies, $\mathrm{p}=0.964)$, TC ( 11 studies, $\mathrm{p}=0.567)$ and TBIL (6

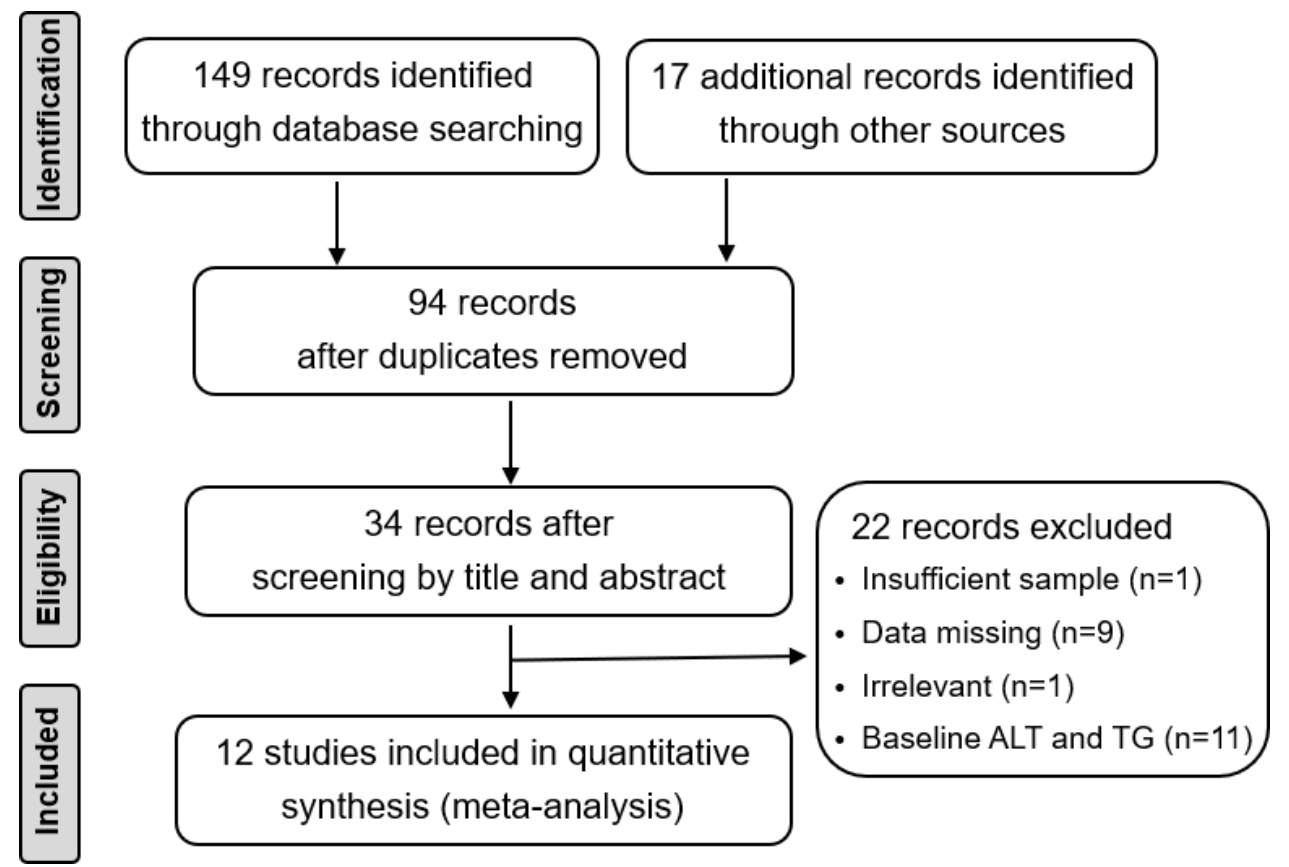

Figure 1 Flow diagram of data selection process. ALT, alanine transaminase; TG, triglyceride. 


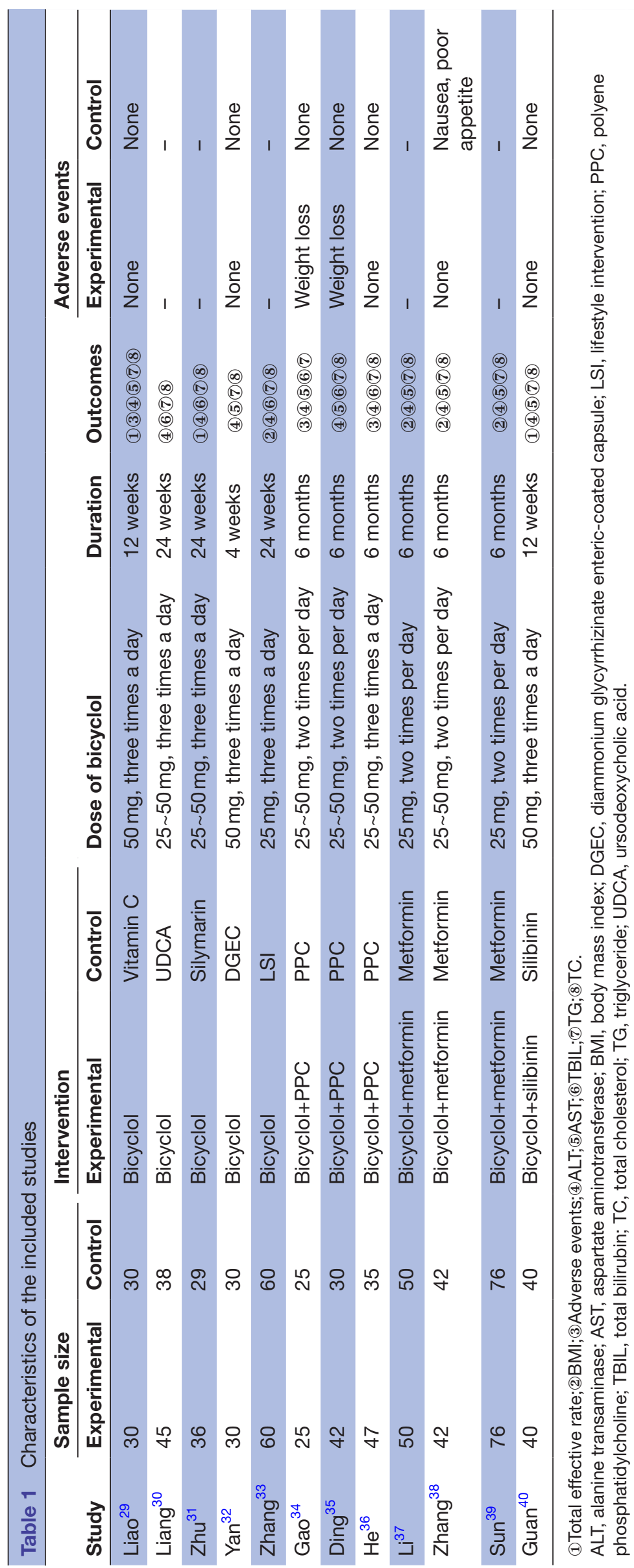



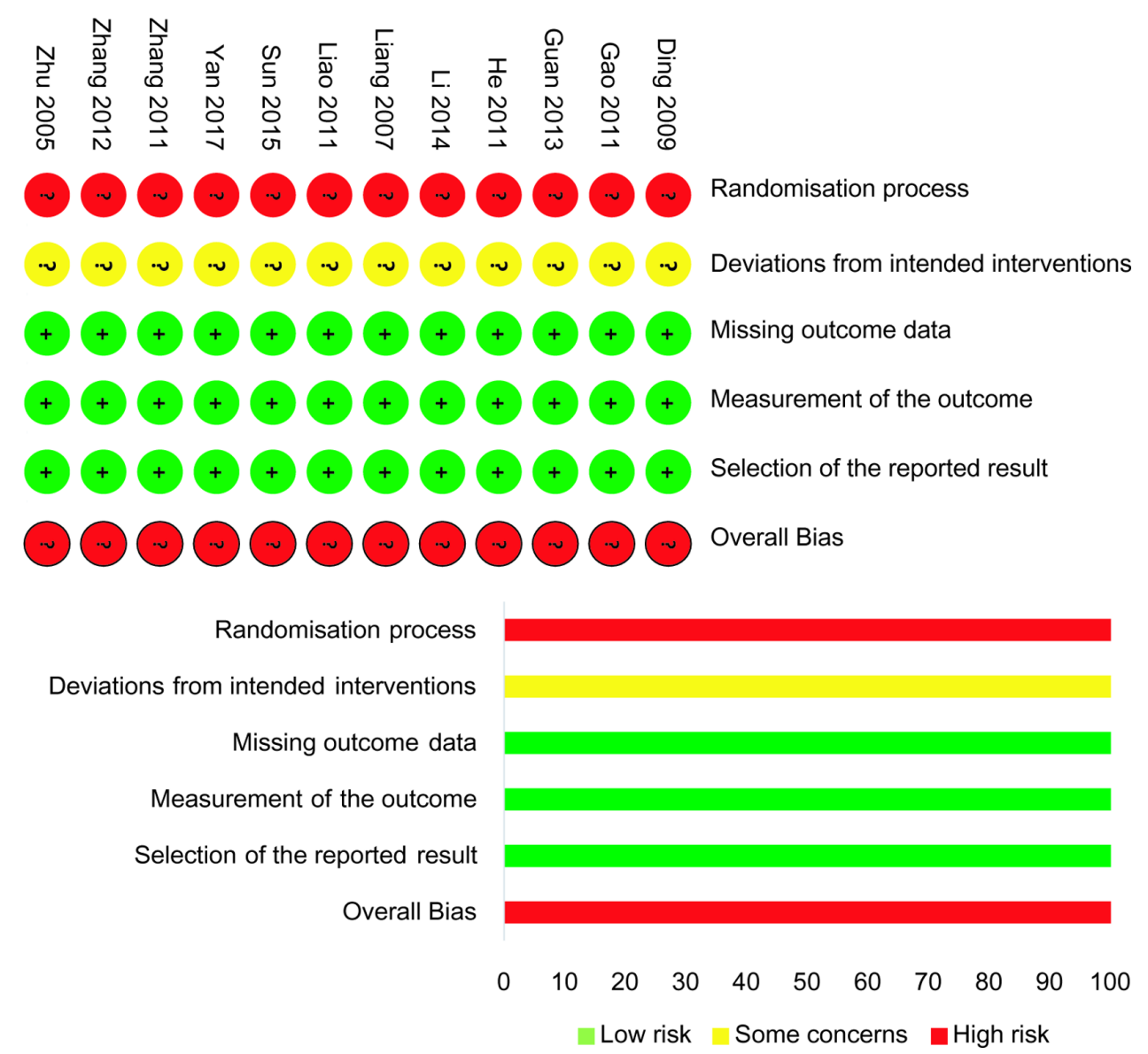

Figure 2 The quality assessment of the included studies. The quality of randomised controlled trials was assessed as a 'high risk', 'low risk' or 'some concerns' to each item independently by two reviewers according to the most recently revised Cochrane risk of bias tool.

studies, $\mathrm{p}=0.485$ ). However, ALT (12 studies, $\mathrm{p}=0.027$ ) and TG (12 studies, $\mathrm{p}=0.004$ ) showed significant publication bias. We speculated that the heterogeneity in the studies was the main determining factor, and a subgroup analysis was conducted.

\section{Effect and safety of the bicyclol intervention for patients with NAFLD}

The therapeutic effect and safety of bicyclol for NAFLD were first evaluated. As shown in figure 3, changes in BMI and the total effective rate at improving fatty liver indicated no heterogeneity, with $\mathrm{I}^{2}$ of $0 \%, \mathrm{p}=0.75$, and $\mathrm{I}^{2}$ of $42 \%, \mathrm{p}=0.18$, respectively. Two hundred and five patients in three studies were included in the analysis of the total effective rate, while 456 patients in four studies were included in the BMI analysis. The fixed-effects model revealed an increased total effective rate (total effective rate: $\mathrm{OR}=4.49 ; 95 \%$ CI 2.02 to $9.95 ; \mathrm{p}=0.0002)$ but no significant effect on BMI (BMI: MD $=-0.68 ; 95 \%$ CI -1.37 to $0.02 ; \mathrm{p}=0.06$ ) in the bicyclol group compared with the control group. No gastrointestinal adverse events, such as nausea, vomiting and diarrhoea, or headache were reported in the bicyclol treatment group in the included studies (table 1).

\section{Effect of bicyclol on liver function biomarkers in patients with NAFLD}

Serum ALT levels were reported in 12 studies. These trials involved 1008 patients, with 523 patients in the treatment group and 485 patients in the control group. A high level of statistical heterogeneity for ALT levels was observed, with $\mathrm{I}^{2}$ of $95 \%$ and $\mathrm{p}<0.00001$. Therefore, we further divided these studies into a bicyclol monotherapy subgroup and bicyclol combination treatment subgroup according to the drug regimen used in the experimental group. ALT levels in the bicyclol monotherapy subgroup, which were analysed using a random-effects model, were significantly decreased compared with those of the corresponding control group (ALT U/L: MD $=-34.07 ; 95 \%$ CI -36.70 to $-31.43 ; \mathrm{p}<0.00001)$. However, significant heterogeneity was observed in the bicyclol combination subgroup with $\mathrm{I}^{2}$ of $95 \%$ and $\mathrm{p}<0.00001$. Therefore, we performed a descriptive analysis and showed that bicyclol was more likely to decrease the levels of ALT in all seven studies when administered in combination with other drugs (figure 4A).

Serum AST levels were recorded in eight trials covering 658 patients, including 335 and 323 participants in the treatment and control groups, respectively. Heterogeneity 
A. Total effective rate

\begin{tabular}{|c|c|c|c|c|c|c|c|c|c|c|c|}
\hline \multirow[b]{2}{*}{ Study or Subgroup } & \multicolumn{2}{|c|}{ Experimental } & \multicolumn{2}{|c|}{ Control } & \multirow[b]{2}{*}{ Weight } & \multirow{2}{*}{$\begin{array}{c}\text { Odds Ratio } \\
\text { M-H, Fixed, } 95 \% \mathrm{Cl}\end{array}$} & \multirow{2}{*}{\multicolumn{4}{|c|}{$\begin{array}{c}\text { Odds Ratio } \\
\text { M-H, Fixed, } 95 \% \mathrm{Cl}\end{array}$}} & \\
\hline & Events & Total & Events & Total & & & & & & & \\
\hline Guan 2013 & 38 & 40 & 35 & 40 & $28.9 \%$ & $2.71[0.49,14.90]$ & & & & & \\
\hline Liao 2011 & 26 & 30 & 11 & 30 & $24.3 \%$ & $11.23[3.10,40.71]$ & & & & & \\
\hline Total $(95 \% \mathrm{Cl})$ & & 106 & & 99 & $100.0 \%$ & $4.49[2.02,9.95]$ & & & & & \\
\hline Total events & 96 & & 69 & & & & & & & & \\
\hline
\end{tabular}

B. BMI

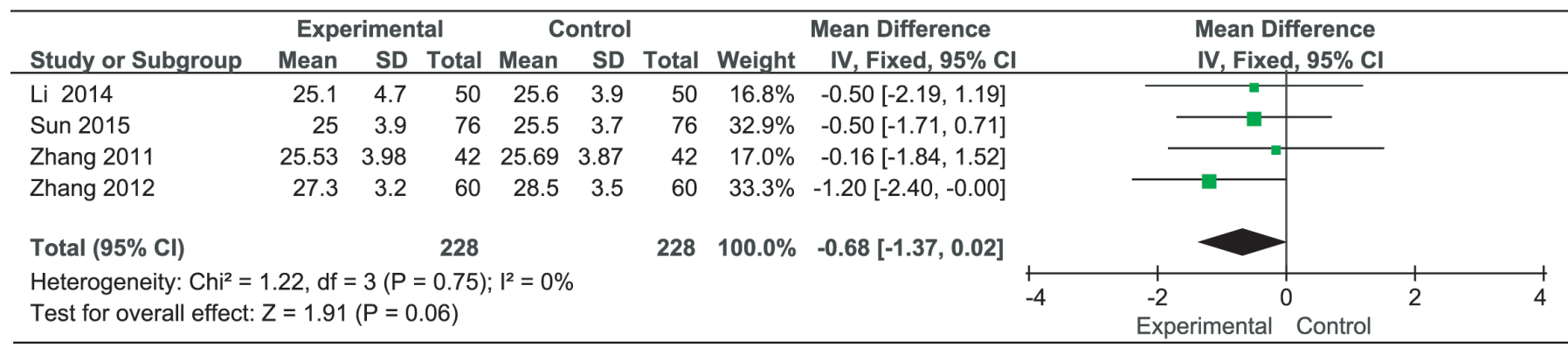

Figure 3 The effect of bicyclol on total effective rate and BMI in patients with NAFLD. Review Manager V.5.3 software was used to analyse the data. OR with its $95 \% \mathrm{Cl}$ was estimated for total effective rate. Mean difference (MD) with its $95 \% \mathrm{Cl}$ was estimated for BMI. Heterogeneities were evaluated using the $\chi^{2}$ and $\mathrm{I}^{2}$ statistics. $\mathrm{I}^{2}<50 \%$ and $\mathrm{p}>0.10$ were deemed as homogeneous and the fixed-effects model was used. $p<0.05$ was considered as statistically different between the experimental and control groups. BMI, body mass index; NAFLD, non-alcoholic fatty liver disease.

was observed for AST levels, with $\mathrm{I}^{2}$ of $74 \%$ (figure $4 \mathrm{~B}$ ). The random-effects model demonstrated that the reduction of AST levels was significant in patients with NAFLD treated by bicyclol as a monotherapy and combination therapy (AST U/L: MD $=-15.20 ; 95 \%$ CI -20.51 to -9.90 ; $\mathrm{p}<0.00001)$.

Serum TBIL levels were detected in six trials, involving 472 participants, with 255 and 217 patients in the treatment and control groups, respectively (figure 4C). There was excellent homogeneity among the six studies, with $\mathrm{I}^{2}=0 \%$ and $\mathrm{p}=0.60$, and the fixed-effects model indicated that bicyclol significantly decreased the TBIL level in patients with NAFLD (TBIL $\mu \mathrm{mol} / \mathrm{L}: \mathrm{MD}=-1.72 ; 95 \% \mathrm{CI}$ -2.72 to $-0.72 ; \mathrm{p}=0.0008)$.

\section{Effect of bicyclol on blood lipid biomarkers in patients with NAFLD}

Twelve studies reported the TG levels. These trials involved 1008 patients, with 523 patients in the treatment groups and 485 patients in the control groups. A high level of statistical heterogeneity was observed for TG levels, with $\mathrm{I}^{2}$ of $90 \%$ and $\mathrm{p}<0.00001$, and thus the subgroup analysis was conducted. The bicyclol combination subgroup did not display heterogeneity, with $\mathrm{I}^{2}=0 \%$ and $\mathrm{p}=0.89$, and it significantly decreased the TG level in patients with NAFLD compared with patients receiving monotherapy with other drugs, which was analysed by a random-effects model (TG mmol/L: MD $=-0.39 ; 95 \%$ p<0.00001). Substantial heterogeneity was observed in the bicyclol monotherapy subgroup, with $\mathrm{I}^{2}$ of $95 \%$ and $\mathrm{p}<0.00001$.
The descriptive analysis showed that bicyclol monotherapy was more likely to decrease the levels of TG in all the five monotherapy studies (figure 5A).

Eleven studies reported the TG levels. These trials involved 958 patients, with 498 and 460 patients in the treatment and control groups, respectively. The $\mathrm{I}^{2}$ of TC was $67 \%$, and therefore, the random-effects model was conducted and showed that the reduction of TC levels in patients with NAFLD treated by bicyclol was significant $(\mathrm{TC} \mathrm{mmol} / \mathrm{L}$ : $\mathrm{MD}=-0.52 ; 95 \% \mathrm{CI}-0.70$ to -0.34 ; $\mathrm{p}<0.00001$ ) (figure 5B).

\section{Grading the evidence}

The evidence for the key outcomes was graded based on the limitations of precision, publication bias, risk of bias and heterogeneity. The quality of evidence was either low or very low (table 2 ).

\section{DISCUSSION}

By performing a meta-analysis of 12 Chinese studies including 1008 patients, this review provided evidence that bicyclol, regardless of its application as a monotherapy or in combination with other drugs, exerts a positive effect on improving liver function (ALT, AST and TBIL) and blood lipid levels (TG and TC). Although the bicyclol combination treatment for ALT levels and monotherapy for TG levels showed considerable heterogeneity, each trial among the included studies reported promising therapeutic effects on abnormal blood biomarker levels. 


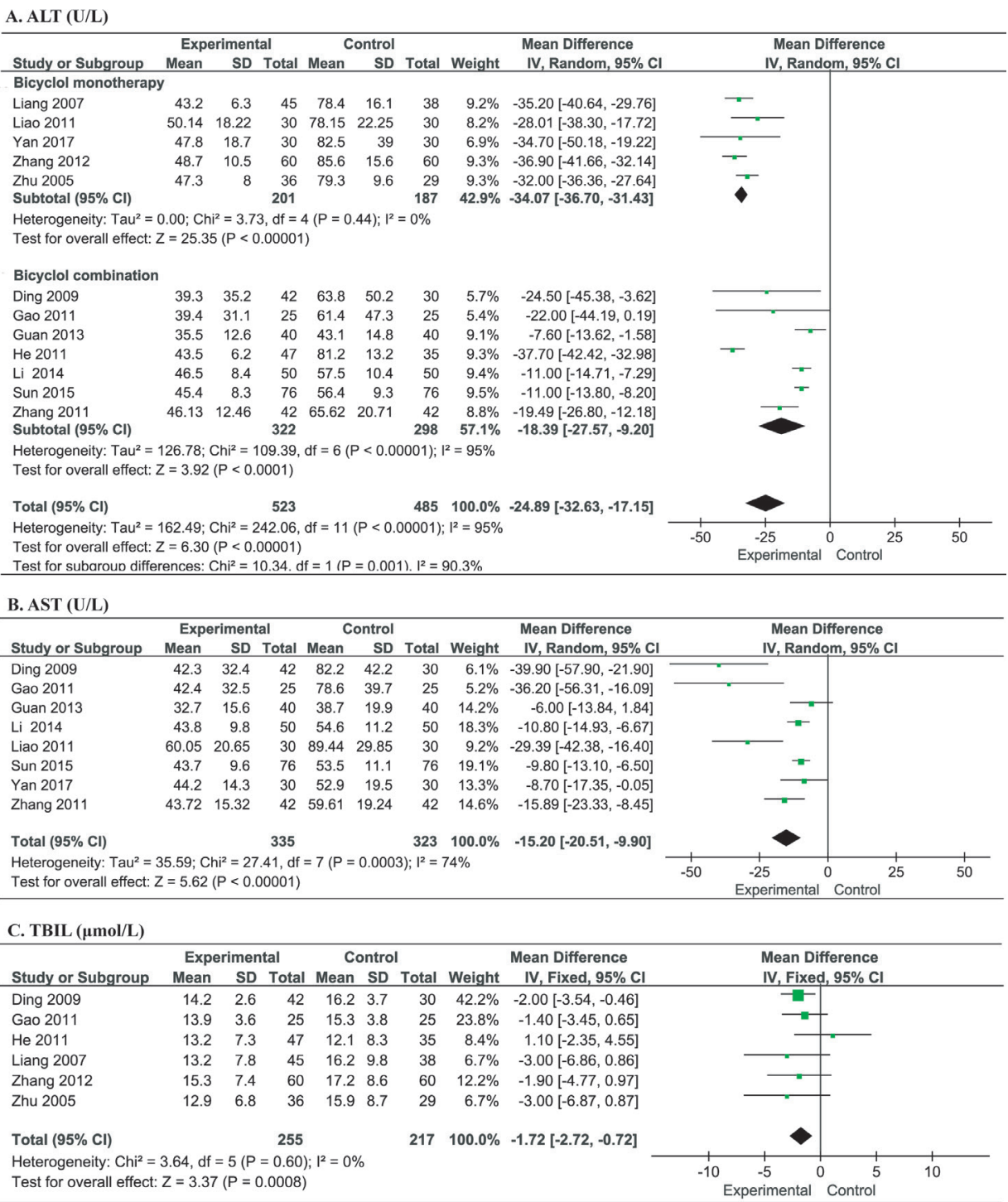

Figure 4 The effect of bicyclol on ALT, AST and TBIL levels in patients with NAFLD. Review Manager V.5.3 software was used to analyse the data. Mean difference (MD) with its $95 \% \mathrm{Cl}$ was estimated for continuous outcomes. Heterogeneities were evaluated using the $\chi^{2}$ and $\mathrm{I}^{2}$ statistics. The ALT parameter was significantly heterogeneous $\left(\mathrm{I}^{2} \geq 75 \%\right.$ and $\left.\mathrm{P}<0.10\right)$ and subgroup analysis was conducted (A); the AST parameter was considered heterogeneous $\left(50 \% \leq \mathrm{I}^{2}<75 \%\right)$ and the random-effects model was used (B); the TBIL parameter was homogeneous $\left(I^{2}<50 \%\right.$ and $\left.p>0.10\right)$ and the fixed-effects model was used (C). $\mathrm{p}<0.05$ was considered as statistically different between the experimental and control groups. ALT, alanine transaminase; AST, aspartate aminotransferase; NAFLD, non-alcoholic fatty liver disease; TBIL, total bilirubin.

In the clinic, bicyclol is recommended for oral administration for up to 6 months. Although adverse events, such as gastrointestinal intolerance were sporadically reported in the control group in this meta-analysis (table 1), these mild discomforts were not reported in the bicyclol-treated group, which agreed with the extremely mild and rare incidence of adverse reactions observed in long-term clinical practice. ${ }^{13}$ Moreover, only three of the included studies concluded that the bicyclol intervention produced a higher total effective rate for fatty liver, which was mainly based on blood biomarker levels and B-model ultrasonography results. We thus evaluated the liver function and blood lipid biomarkers as the primary outcome, although liver histology is the gold standard and MRI has higher accuracy for assessing fatty liver. ${ }^{17}$
The pathogenesis of NAFLD is complex and is strongly associated (over $76 \%$ ) with T2DM; ${ }^{41-43}$ patients with or without T2DM were thus included in this review. Additionally, the course of the disease varied among the included studies, and some studies did not report the patient's medical history; therefore, we limited the baseline ALT and TG levels to ensure the consistency of the included patients as much as possible. We also defined the treatment duration as at least 4 weeks, because NAFLD is a chronic disease and bicyclol is suitable for long-term oral administration. Although the use of bicyclol to treat NAFLD is an off-label use, the Chinese guidelines of prevention and treatment for NAFLD updated in 2018 recommend that hepatoprotectants are potentially complementary treatment measures for patients 
A. TG (mmol/L)

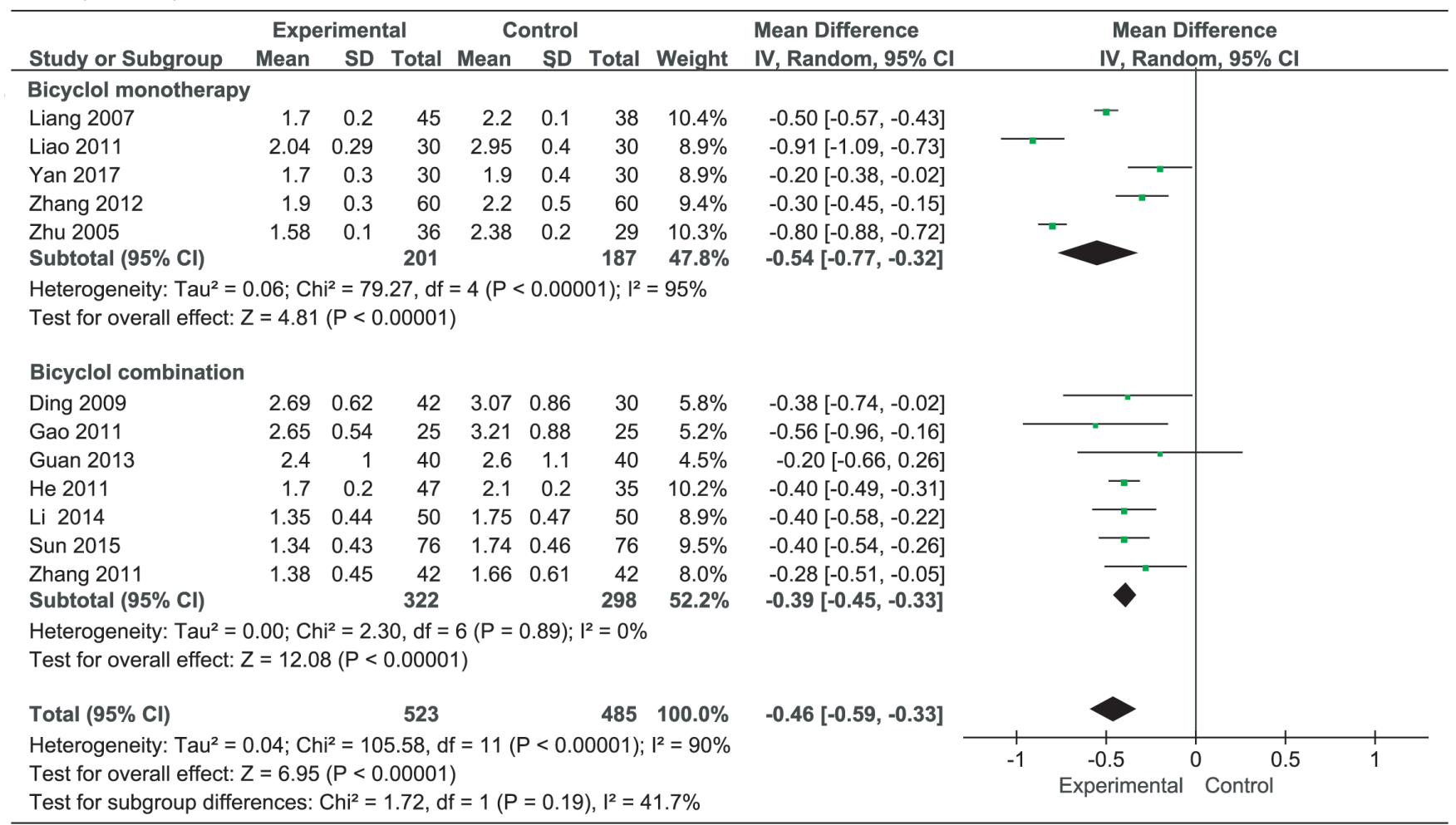

B. TC $(\mathrm{mmol} / \mathrm{L})$

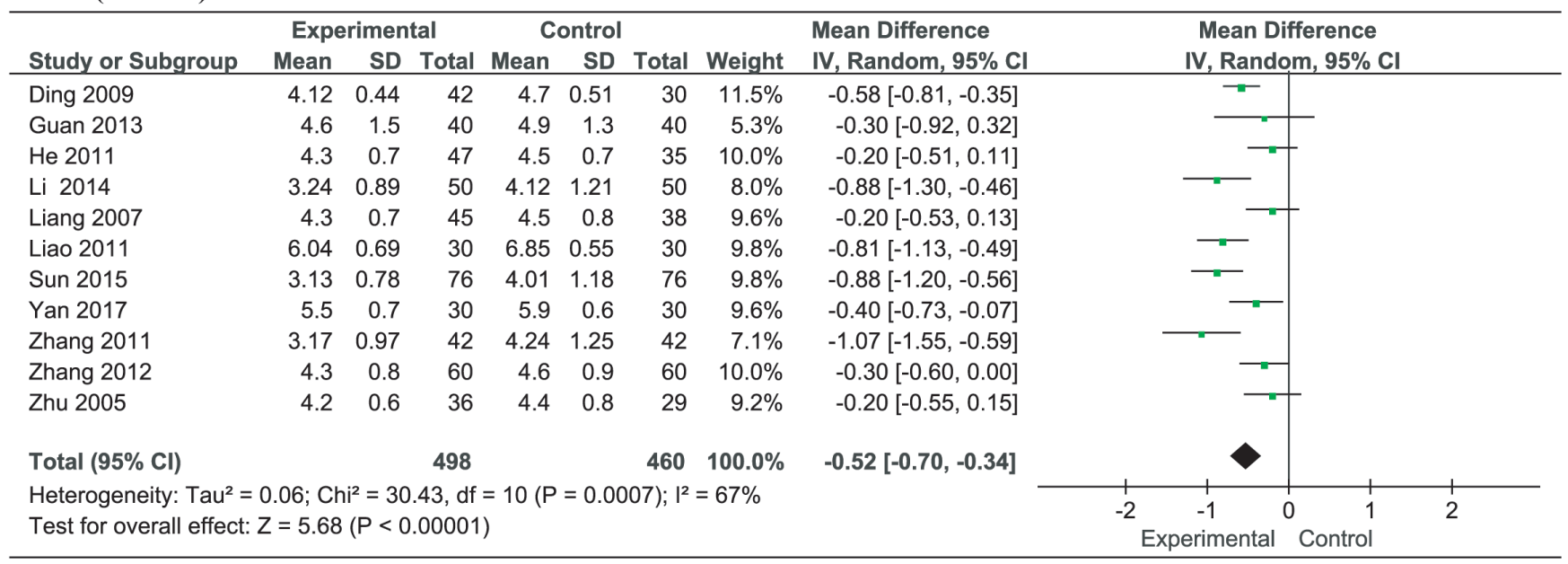

Figure 5 The effect of bicyclol on TG and TC levels in patients with NAFLD. Review Manager V.5.3 software was used to analyse the data. Mean difference (MD) with its $95 \% \mathrm{Cl}$ was estimated for continuous outcomes. Heterogeneities were evaluated using the $\chi^{2}$ and $I^{2}$ statistics. The TG parameter was significantly heterogeneous $\left(I^{2} \geq 75 \%\right)$ and subgroup analysis was conducted (A); the TC parameter was considered heterogeneous $\left(50 \% \leq I^{2}<75 \%\right)$ and the random-effects model was used (B). $p<0.05$ was considered as statistically different between the experimental and control groups. NAFLD, alcoholic fatty liver disease; TC, total cholesterol; TG, triglyceride.

with NASH with elevated aminotransferase levels or liver injury. ${ }^{44}$ Compared with the intervention in the control group, including lifestyle changes and other drug treatments, the alleviation of abnormal blood biomarker levels by bicyclol is evident and consistent with its clinical practice. ${ }^{21}$ Notably, subgroup analyses for ALT and TG levels, which were conducted when significant heterogeneity existed, also provided substantial evidence for its effect.

This review has to interpret the limitations of the low quality of the included studies, publication bias and low grading of evidence. All the included studies were conducted in China, and many of them did not provide a description of specific methods of blinding and random allocation concealments. In terms of the outcome indicators, most articles lacked information on the blood glucose levels and insulin resistance index, and thus the results of the meta-analysis merely provide the effect of bicyclol on liver function and blood lipid indicators. Though the biomarkers AST, TC and TBIL showed no publication bias, ALT and TG showed significant 


\begin{tabular}{|c|c|c|c|c|}
\hline Outcomes & Corresponding risk $(95 \% \mathrm{Cl})^{*}$ & $\begin{array}{l}\text { No. of participants } \\
\text { (studies) }\end{array}$ & $\begin{array}{l}\text { Quality of the } \\
\text { evidence } \\
\text { (GRADE) }\end{array}$ & Comments \\
\hline ALT & $\begin{array}{l}\text { The mean ALT in the intervention groups was } 24.89 \\
\text { SD lower ( } 32.63 \text { to } 17.15 \text { lower) }\end{array}$ & $\begin{array}{l}1008 \\
\text { (12 studies) }\end{array}$ & $\begin{array}{l}\oplus \ominus \ominus \ominus \text { very } \\
\text { low†‡ }\end{array}$ & $\begin{array}{l}\text { SMD }-24.89 \\
(-32.63 \text { to }-17.15)\end{array}$ \\
\hline AST & $\begin{array}{l}\text { The mean AST in the intervention groups was } 15.2 \\
\text { SD lower ( } 20.51 \text { to } 9.9 \text { lower) }\end{array}$ & $\begin{array}{l}658 \\
\text { (eight studies) }\end{array}$ & $\oplus \oplus \ominus \ominus$ low $\neq$ & $\begin{array}{l}\text { SMD }-15.2 \\
(-20.51 \text { to }-9.9)\end{array}$ \\
\hline TBIL & $\begin{array}{l}\text { The mean TBIL in the intervention groups was } 1.72 \\
\text { SD lower ( } 2.72 \text { to } 0.72 \text { lower) }\end{array}$ & $\begin{array}{l}472 \\
\text { (six studies) }\end{array}$ & $\oplus \oplus \ominus \ominus$ low $\neq$ & $\begin{array}{l}\text { SMD }-1.72 \\
(-2.72 \text { to }-0.72)\end{array}$ \\
\hline TG & $\begin{array}{l}\text { The mean TG in the intervention groups was } 0.46 \text { SD } \\
\text { lower ( } 0.59 \text { to } 0.33 \text { lower) }\end{array}$ & $\begin{array}{l}1008 \\
\text { (12 studies) }\end{array}$ & $\begin{array}{l}\bigoplus \ominus \ominus \ominus \text { very } \\
\text { low†‡ }\end{array}$ & $\begin{array}{l}\text { SMD }-0.46 \\
(-0.59 \text { to }-0.33)\end{array}$ \\
\hline TC & $\begin{array}{l}\text { The mean TC in the intervention groups was } 0.52 \mathrm{SD} \\
\text { lower ( } 0.7 \text { to } 0.34 \text { lower) }\end{array}$ & $\begin{array}{l}958 \\
\text { (11 studies) }\end{array}$ & $\bigoplus \oplus \ominus \ominus$ low $\neq$ & $\begin{array}{l}\text { SMD }-0.52 \\
(-0.7 \text { to }-0.34)\end{array}$ \\
\hline
\end{tabular}

GRADE Working Group grades of evidence.

High quality: Further research is very unlikely to change our confidence in the estimate of effect.

Moderate quality: Further research is likely to have an important impact on our confidence in the estimate of effect and may change the estimate.

Low quality: Further research is very likely to have an important impact on our confidence in the estimate of effect and is likely to change the estimate.

Very low quality: We are very uncertain about the estimate.

*The corresponding risk (and its $95 \% \mathrm{Cl}$ ) is based on the assumed risk in the comparison group and its $95 \% \mathrm{Cl}$. †Downgraded one level due to serious limitations in publication bias.

‡Downgraded one level for including studies with high risk of bias.

ALT, alanine transaminase; AST, aspartate aminotransferase; GRADE, Grading of Recommendations Assessment, Development and Evaluation; SMD, standardised mean difference; TBIL, total bilirubin; TC, total cholesterol; TG, triglyceride.

publication bias. We speculated that the heterogeneity and language bias contributed to this publication bias, and subgroup analysis was conducted. Additionally, when the degree of heterogeneity was large, Egger's tests did not have good properties. ${ }^{45}$ Similarly, the low grading of evidence was mainly derived from the publication bias, risk of bias and heterogeneity. Therefore, the results of the meta-analysis merely provide a reference based on the current evidence.

In conclusion, the present study presents the effectiveness of bicyclol monotherapy and/or combination therapy at ameliorating the altered liver function and blood lipid biomarkers in patients with NAFLD. This preliminary study predicts that bicyclol might be an alternative available drug to be explored for NAFLD therapy in the future. However, the conclusion also needs to be further verified in more well-designed and implemented studies.

Contributors Conceptualisation, analysis, writing original draft, visualisation: $\mathrm{HL}$; Validation of data and analysis: N-NL; Supervision, validation and writing draft: Z-GP; Approval of final manuscript: all authors.

Funding This work was supported by CAMS Innovation Fund for Medical Sciences (2019-I2M-1-001), National Natural Science Foundation, China (81621064), and National Mega-Project for 'R\&D for Innovative drugs', Ministry of Science and Technology, China (2018ZX09711001-003-010).

Competing interests None declared

Patient consent for publication Not required.

Provenance and peer review Not commissioned; externally peer reviewed.

Data availability statement All data relevant to the study are included in the article or uploaded as supplementary information.
Supplemental material This content has been supplied by the author(s). It has not been vetted by BMJ Publishing Group Limited (BMJ) and may not have been peer-reviewed. Any opinions or recommendations discussed are solely those of the author(s) and are not endorsed by BMJ. BMJ disclaims all liability and responsibility arising from any reliance placed on the content. Where the content includes any translated material, BMJ does not warrant the accuracy and reliability of the translations (including but not limited to local regulations, clinical guidelines, terminology, drug names and drug dosages), and is not responsible for any error and/or omissions arising from translation and adaptation or otherwise.

Open access This is an open access article distributed in accordance with the Creative Commons Attribution Non Commercial (CC BY-NC 4.0) license, which permits others to distribute, remix, adapt, build upon this work non-commercially, and license their derivative works on different terms, provided the original work is properly cited, appropriate credit is given, any changes made indicated, and the use is non-commercial. See: http://creativecommons.org/licenses/by-nc/4.0/.

ORCID iD

Hu Li http://orcid.org/0000-0002-7609-9399

\section{REFERENCES}

1 Friedman SL, Neuschwander-Tetri BA, Rinella M, et al. Mechanisms of NAFLD development and therapeutic strategies. Nat Med 2018;24:908-22.

2 Rosso N, Bellentani S. Nonalcoholic Fatty Liver Disease: A Wide Spectrum Disease. In: Radu-lonita F, Pyrsopoulos N, Jinga M, et al, eds. Liver diseases. Springer, Cham, 2020.

3 Shi T, Wu L, Ma W, et al. Nonalcoholic fatty liver disease: pathogenesis and treatment in traditional Chinese medicine and Western medicine. Evid Based Complement Alternat Med 2020;2020:1-16.

4 Younossi ZM, Koenig AB, Abdelatif D, et al. Global epidemiology of nonalcoholic fatty liver disease-Meta-analytic assessment of prevalence, incidence, and outcomes. Hepatology 2016;64:73-84.

$5 \mathrm{Li} \mathrm{J}$, Zou B, Yeo YH, et al. Prevalence, incidence, and outcome of non-alcoholic fatty liver disease in Asia, 1999-2019: a systematic review and meta-analysis. Lancet Gastroenterol Hepatol 2019;4:389-98. 
6 Koutoukidis DA, Astbury NM, Tudor KE, et al. Association of weight loss interventions with changes in biomarkers of nonalcoholic fatty liver disease: a systematic review and meta-analysis. JAMA Intern Med 2019;179:1262-71.

7 Sridharan K, Sivaramakrishnan G, Sequeira RP, et al. Pharmacological interventions for non-alcoholic fatty liver disease: a systematic review and network meta-analysis. Postgrad Med J 2018;94:556-65

8 Katsagoni CN, Georgoulis M, Papatheodoridis GV, et al. Effects of lifestyle interventions on clinical characteristics of patients with non-alcoholic fatty liver disease: a meta-analysis. Metabolism 2017;68:119-32.

9 Hannah WN, Harrison SA. Lifestyle and dietary interventions in the management of nonalcoholic fatty liver disease. Dig Dis Sci 2016;61:1365-74.

10 Boeckmans J, Natale A, Rombaut M, et al. Anti-NASH drug development Hitches a lift on PPAR agonism. Cells 2019;9:E37

11 Muthiah MD, Sanyal AJ. Current management of non-alcoholic steatohepatitis. Liver Int 2020;40 Suppl 1:89-95.

12 Younossi ZM, Loomba R, Rinella ME, et al. Current and future therapeutic regimens for nonalcoholic fatty liver disease and nonalcoholic steatohepatitis. Hepatology 2018;68:361-71.

13 Liu GT. Bicyclol: a novel drug for treating chronic viral hepatitis B and C. Med Chem 2009;5:29-43.

$14 \mathrm{Li} \mathrm{H}$, Huang MH, Jiang JD, et al. Hepatitis C: from inflammatory pathogenesis to anti-inflammatory/hepatoprotective therapy. World $J$ Gastroenterol 2018;24:5297-311.

15 Li H, Li JR, Huang MH, et al. Bicyclol Attenuates Liver Inflammation Induced by Infection of Hepatitis C Virus via Repressing ROSMediated Activation of MAPK/NF-кB Signaling Pathway. Front Pharmacol 2018;9:1438.

16 Huang $\mathrm{MH}$, Li H, Xue R, et al. Up-Regulation of glycolipid transfer protein by bicyclol causes spontaneous restriction of hepatitis $\mathrm{C}$ virus replication. Acta Pharm Sin B 2019;9:769-81.

17 Lee SS, Park SH. Radiologic evaluation of nonalcoholic fatty liver disease. World J Gastroenterol 2014;20:7392-402.

18 Wong VW-S, Adams LA, de Lédinghen V, et al. Noninvasive biomarkers in NAFLD and NASH - current progress and future promise. Nat Rev Gastroenterol Hepatol 2018;15:461-78.

19 Vilar-Gomez E, Chalasani N. Non-Invasive assessment of nonalcoholic fatty liver disease: clinical prediction rules and blood-based biomarkers. J Hepatol 2018;68:305-15.

20 Yu HY, Wang BL, Zhao J, et al. Protective effect of bicyclol on tetracycline-induced fatty liver in mice. Toxicology 2009;261:112-8.

21 Han Y, Shi JP, Ma AL, et al. Randomized, vitamin E-controlled trial of bicyclol plus metformin in non-alcoholic fatty liver disease patients with impaired fasting glucose. Clin Drug Investig 2014;34:1-7.

22 Cumpston M, Li T, Page MJ, et al. Updated guidance for trusted systematic reviews: a new edition of the Cochrane Handbook for systematic reviews of interventions. Cochrane Database Syst Rev 2019;10:ED000142.

23 Moher Det al. Preferred reporting items for systematic reviews and meta-analyses: the PRISMA statement. Ann Intern Med 2009;151:264-9.

24 Martin-Rodriguez JL, Gonzalez-Cantero J, Gonzalez-Cantero A, et al. Diagnostic accuracy of serum alanine aminotransferase as biomarker for nonalcoholic fatty liver disease and insulin resistance in healthy subjects, using 3T MR spectroscopy. Medicine 2017;96:e6770.

25 Higgins JPT, Savović J, Page MJ, et al. Chapter 8: Assessing risk of bias in a randomized trial. In: Higgins JPT, Thomas J, Chandler $\mathrm{J}$, et al, eds. Cochrane Handbook for systematic reviews of interventions version 6.0. Cochrane, 2019.

26 Wei X, Wang C, Hao S, et al. The therapeutic effect of berberine in the treatment of nonalcoholic fatty liver disease: a meta-analysis. Evid Based Complement Alternat Med 2016;2016:1-9.
27 Turner RM, Davey J, Clarke MJ, et al. Predicting the extent of heterogeneity in meta-analysis, using empirical data from the Cochrane database of systematic reviews. Int J Epidemiol 2012;41:818-27.

28 Sridharan K, Sequeira RP. Drugs for treating severe hypertension in pregnancy: a network meta-analysis and trial sequential analysis of randomized clinical trials. Br J Clin Pharmacol 2018;84:1906-16.

29 Liao B. Efficacy of bicyclol in the treatment of nonalcoholic fatty liver disease. Capital medicine 2011;18:42-3.

30 Liang SG, Liu WD, Wang JF. Clinical study in the treatment of nonalcoholic steatohepatitis with bicyclol tablets. J Clin Hepatol 2007;23:375-6.

31 Zhu Y. Treatment of non-alcoholic steatohepatitis (NASH) with bicyclol tablets. World Chinese Journal of Digestology 2005;13:139-40.

32 Yan X. Clinical efficacy of nonalcoholic steato-hepatitis treated by bicyclol. In: Guide of China medicine. 15, 2017.

33 Zhang Z. Efficacy observation of bicyclol tablets in the treatment of non-alcoholic steatohepatitis. Capital Medicine 2012;19:34-5.

34 Gao D. Combined effect analysis of two drugs in the treatment of non-alcoholic steatohepatitis. China Medicine and Pharmacy 2011;01:97.

35 Ding MY, Qin XM, Ma J, et al. Effect of polyene phosphatidylcholine combined with bicyclol in the treatment of nonalcoholic steatohepatitis. J Med Pract 2009;25:1289-91.

$36 \mathrm{He} \mathrm{J}$. Effect of bicyclol combined with polyene phosphatidylcholine on nonalcoholic steatohepatitis. Capital Medicine 2011;18:34.

$37 \mathrm{Li} \mathrm{Q}, \mathrm{Wu}$ Y, Hu Q. Efficacy analysis of bicyclol combined with metformin in the treatment of type 2 diabetes mellitus with nonalcoholic fatty liver disease. Laboratory Medicine and Clinic 2014;11:3477-8.

38 Zhang Y, Zeng CY, Tian XN, et al. Effect of metformin combined with bicyclol on liver function and glucose metabolism of type 2 diabetes patients with nonalcoholic fatty liver. J Guiyang Medical Colleage 2011;36:598-600.

39 Sun D. Clinical efficacy observation of 152 nonalcoholic fatty liver disease patients with type 2 diabetes mellitus treated by bicyclol combined with metformin. J China Prescription Drug 2015;13:81-2.

40 Guan Y, YG X, Pan D, et al. Clinical efficacy observation of bicyclo combined with silibinin in the treatment of nonalcoholic fatty hepatitis with type 2 diabetes mellitus. Guide of China medicine 2013;11:606-7.

41 Tamada H, Naito H, Kitamori K, et al. Efficacy of dietary lipid control in healing high-fat and high-cholesterol diet-induced fibrotic steatohepatitis in rats. PLoS One 2016;11:e0145939.

42 Huang M, Kim HG, Zhong X, et al. Sestrin 3 protects against dietinduced nonalcoholic steatohepatitis in mice through suppression of transforming growth factor $\beta$ signal transduction. Hepatology 2020;71:76-92.

43 Liu Z, Zhang Y, Graham S, et al. Causal relationships between NAFLD, T2D and obesity have implications for disease subphenotyping. J Hepatol 2020;73:263-76.

44 Committee of Hepatology, Chinese Research Hospital Association, Fatty Liver Expert Committee, Chinese Medical Doctor Association, National Workshop on Fatty Liver and Alcoholic Liver Disease, Chinese Society of Hepatology. Expert recommendations on standardized diagnosis and treatment for fatty liver disease in China (2019 revised edition. Zhonghua Gan Zang Bing Za Zhi 2019;27:748-53.

45 Muka T, Glisic M, Milic J, et al. A 24-step guide on how to design, conduct, and successfully publish a systematic review and metaanalysis in medical research. Eur J Epidemiol 2020;35:49-60. 